\title{
Estimación del período de construcción de las edificaciones del cantón de San José por medio de análisis de fotografías aéreas
}

\author{
Estimation of the construction period of the buildings \\ (construction period) in the canton of San José by analysis of \\ aerial photographs
}

\author{
Silvia Blanco-Sáenz ${ }^{1}$. \\ Universidad de Costa Rica, Costa Rica \\ Luis Carlos Esquivel-Salas ${ }^{2}$ \\ Universidad de Costa Rica, Costa Rica
}

\begin{abstract}
Resumen
La fecha de construcción es una característica de suma importancia para las edificaciones, ya que permite, entre varias aplicaciones, el determinar de manera general su desempeño o comportamiento ante terremotos. Este dato es generalmente difícil de obtener para grandes grupos de estructuras, el cual es el caso del cantón de San José. Se presenta entonces un estudio en el cual se estimó un periodo de construcción aproximado para las 84000 edificaciones de San José, analizando fotografías aéreas disponibles en el Instituto Geográfico Nacional (IGN) de Costa Rica de forma manual. Este dato puede ser utilizado para varios fines, entre ellos evaluaciones de riesgo ante amenazas naturales. Se expone su valor y utilidad en el contexto de un estudio de riesgo sísmico físico del cantón de San José en desarrollo por parte del Laboratorio de Ingeniería Sísmica del Instituto de Investigaciones en Ingeniería de la Universidad de Costa Rica. Se encontró que la mayor cantidad de estructuras, en términos absolutos, se construyó en el periodo de 1989-2004, para un total de 18 399 , mientras que la tasa de construcción promedio por año más alta fue de 2675 para el periodo de 1974-1979. Un 38 \% de las edificaciones se construyeron previo a la existencia del Código Sísmico

1 Bachiller en Geografía, de la Universidad de Costa Rica, correo electrónico: silvia.25.10.95@gmail.com (iD https://orcid.org/0000-0002-3583-7412

2 Máster en Ingeniería Estructural, Universidad de Costa Rica. Investigador del Laboratorio de Ingeniería Sísmica (LIS-UCR). Correo electrónico: luiscarlos.esquivel@ucr.ac.cr iD https://orcid.org/0000-0003-4208-6482
\end{abstract}


Silvia Blanco-Sáenz, Luis Carlos Esquivel-Salas Estimation of the construction period of the buildings (construction period) in the canton of San José by analysis of aerial photographs

de Costa Rica (CSCR), un 60 \% utilizando las versiones de CSCR de 1974 y 1986, mientras que solamente un $2 \%$ utilizaron las versiones más modernas del código (2002 y 2010).

Palabras clave: fecha de construcción, fotografías aéreas, cantón de San José, edificaciones.

\begin{abstract}
The construction period is a very important characteristic of buildings, since it allows, among various applications, to determine, in a general way, their performance or behavior in the event of seismic activity. Construction period data is generally difficult to obtain for large groups of structures, as is the case in the canton of San José. A study is thus presented in which the approximate date of construction was estimated for the 84,004 buildings present in the canton of San José by visual non-automated analysis of aerial photographs available from the Instituto Geográfico Nacional $(I G N)$ (translated as National Geographic Institute) of Costa Rica. Construction period data can be used for various purposes including risk assessments of natural hazards. The value and usefulness of the present investigation is exposed in the context of the risk assessment evaluation on physical damage caused by seismic activity in the canton of San José undertaken by the Laboratorio de Ingeniería Sísmica del Instituto de Investigaciones en Ingeniería de la Universidad de Costa Rica (translated as Seismic Engineering Laboratory of the Engineering Research Institute of the University of Costa Rica). In absolute terms, it was found that the majority of structures were built in the period 1989-2004 and amounted to a total of 18,399 buildings; the highest average annual construction rate was $2,675 / \mathrm{yr}$ for the period $1974-1979.38 \%$ of the buildings were built prior to the existence of the Costa Rican Seismic Code (CSCR), $60 \%$ of the buildings adhered to CSCR Codes in its past versions of 1974 and 1986, while only $2 \%$ complied with the newer versions of 2002 and 2010.
\end{abstract}

Keywords: Construction period; Aerial photographs; Canton of San José; Buildings.

\title{
Introducción
}

La fecha de construcción es una característica de suma importancia para las edificaciones, porque permite, por ejemplo, determinar de manera general su desempeño o comportamiento ante terremotos. Esto es especialmente importante en evaluaciones de riesgo sísmico físico, las cuales generalmente se llevan a cabo considerando un gran número de estructuras, del orden de miles. Ejemplos a nivel mundial de estas evaluaciones son Hazards US Multi-Hazard, conocido como HAZUS-MH (FEMA, 2013) y An Advanced Approach to Earthquake Risk Scenarios With Application to Different European Towns, conocido como RISK-UE (Mouroux et al., 2004).

Generalmente, este dato no se encuentra disponible y tampoco es sencillo de obtener o generar para miles de estructuras al mismo tiempo, lo cual conlleva a dos acciones: (1) utilizar periodos de construcción en lugar de fechas de construcción y (2) valorar utilizar métodos alternativos para obtener este dato de manera aproximada. En este trabajo se expone uno de 
estos métodos alternativos, el cual consiste en la estimación del periodo de construcción de edificaciones a partir del análisis de fotografías aéreas y ortofotos, aplicado al cantón de San José.

La estimación del periodo de construcción para las 84000 edificaciones del cantón de San José es una actividad importante del proyecto en desarrollo del Laboratorio de Ingeniería Sísmica del Instituto de Investigaciones en Ingeniería de la Universidad de Costa Rica (LIS-UCR), titulado "Estimación del riesgo sísmico en el cantón de San José, Costa Rica" (Esquivel-Salas, 2020). En este proyecto se consideran los tres componentes necesarios para realizar una evaluación de riesgo sísmico físico, a saber: amenaza, exposición y vulnerabilidad (Simpson et al., 2014).

El LIS-UCR identificó que en evaluaciones anteriores de riesgo sísmico para este cantón (Calderón, 2016; Calderón \& Silva, 2019; Cardona et al., 2009a, 2009b, 2009c, 2009d; Evaluación de Riesgos Naturales y Antropogénicos, 2014; Miyamoto Internacional Inc., 2016), los componentes menos estudiados en nuestro país eran exposición y vulnerabilidad. La vulnerabilidad describe el comportamiento estructural de las edificaciones, su estudio y descripción se salen del alcance de este artículo y se tratarán en artículos posteriores en revistas de las disciplinas correspondientes.

La exposición, a su vez el menos estudiado de estos dos componentes, consiste en la descripción de los bienes expuestos a la amenaza natural, pudiendo incluir, por ejemplo, población, edificaciones, carreteras, tuberías de agua potable, cosechas, entre muchos otros. Es precisamente por esta razón que el LIS-UCR enfoca los esfuerzos del estudio mencionado en el componente de exposición, incluyendo este una descripción estructural de las edificaciones del cantón, la cual se basa en siete atributos clave, uno de ellos siendo el periodo de construcción. Es aquí donde destaca la importancia del poder estimar un periodo de construcción, aunque sea aproximado, para cada una de las 84000 edificaciones de San José.

El contar con este periodo de construcción aproximado para cada edificación permite asignar, con las limitaciones del caso (e.g. asumiendo que la construcción siguió la normativa vigente), la versión del Código Sísmico de Costa Rica (CSCR) aplicable para cada una de ellas. Esto se traduce en una caracterización más precisa de su desempeño estructural ante terremotos, ya que, por ejemplo, las estructuras diseñadas con versiones más recientes del CSCR deberían tener un mejor desempeño que las más antiguas. 


\section{Zona de estudio}

La zona de estudio fue el cantón de San José (ver figura 1), esta es la sede de la capital del país y representa el $0.09 \%$ del territorio y cuenta con 11 distritos. Según la Municipalidad de San José (MSJ, 2011), al año 2011 el uso de las edificaciones se distribuía de la siguiente manera: un $24.65 \%$ a residencias, un $9.77 \%$ a industria y comercio, mientras que el $6.72 \%$ eran áreas verdes con un $7.45 \%$ de zonas con alguna categoría de protección es decir, no urbanizadas, entre otras.

Figura 1. Mapa de ubicación del cantón de San José.

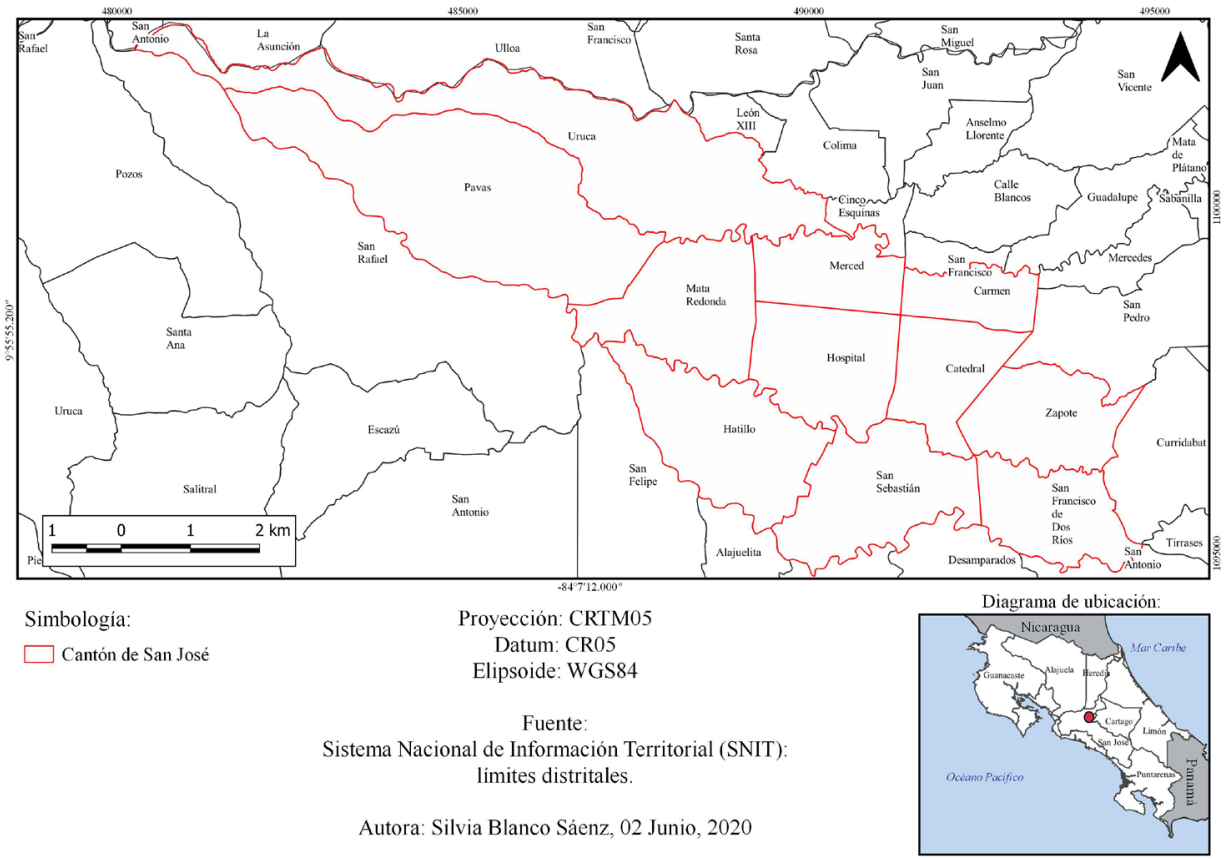

\section{Materiales y Métodos}

Los materiales empleados para la realización de la investigación fueron una serie de fotografías aéreas y mosaicos de ortofotos del cantón de San José brindadas por el Instituto Geográfico Nacional (IGN) de Costa Rica, que cuenta con una serie de archivos fotográficos de todo el país. Previo al año 1989 solo se hallan fotos aéreas, sin embargo para los siguientes 
años, existe una serie de proyectos de mosaicos de ortofotos realizados por la misma institución. Las fotos aéreas utilizadas se describen en la tabla 1:

Tabla 1. Fotografías aéreas utilizadas.

\begin{tabular}{|l|c|c|c|}
\hline Año & Proyecto & Escala & Fotos \\
\hline $\mathbf{1 9 4 5}$ & $7324 \mathrm{M}$ & $1: 40000$ & $126,128,130$. \\
\hline $\mathbf{1 9 6 3}$ & AF61-8 & $1: 20000$ & $430,432,434,436,471,473,475$. \\
\hline $\mathbf{1 9 7 4}$ & Puriscal - Parrita & $1: 30000$ & $102,111,119,205,210$. \\
\hline $\mathbf{1 9 8 0}$ & La Guácima - Tres Ríos & $1: 35000$ & $21952,21954,21956,21958$. \\
\hline $\mathbf{1 9 8 9}$ & Jica & $1: 20000$ & $992,996,1177,1179,1181,1183,1185$. \\
\hline $\mathbf{2 0 0 5}$ & PRCR & $1: 25000$ & $39,40,41,42,43,44$. \\
\hline $\mathbf{2 0 1 4}$ & Actualización & $1: 6000$ & $\begin{array}{l}337,338,339,340,341,342,343,344, \\
345,346,347,272,273,274,275,276, \\
\end{array}$ \\
& & & $277,278,279,280,281,282$. \\
\hline
\end{tabular}

Fuente: IGN.

Además, el Sistema Nacional de Información Territorial (SNIT) cuenta con un una serie de servicios Open Geospatial Consortium (OGC), entre los que se halla un proyecto de mosaico de ortofotos de vuelos realizados entre los años 2014 al 2017, el mismo se utilizó para clasificar los polígonos correspondientes al año de estudio 2017.

También, se contó con una capa shape brindada por la Municipalidad de San José (MSJ), que contenía los polígonos de las huellas constructivas de las edificaciones del cantón. Es capa se generó con base en fotografías aéreas del Proyecto de Catastro Municipal del año 2002, se creó un mapa fotogramétrico con las ortofotos y a partir de ahí se generaron las huellas constructivas. La capa ha sido actualizada pero en general la base se mantiene (Obando, 2018). Finalmente, se utilizó la herramienta $Q$ Geographic Information System (QGIS), la cual es un software libre y de código abierto para la visualización y manejo de datos (Lizano, 2017, p. 3).

\section{Depuración de la capa shape}

Una vez obtenida la capa shape es necesario su respectiva depuración, esto significa la realización de una revisión cruzada entre las edificaciones pertenecientes al shape de la MSJ y la versión más reciente de las ortofotos del SNIT. En este proceso los mayores arreglos corresponden 
a la creación de polígonos debido a que el original abarcaba más de una edificaciones, se eliminaron estructuras demolidas, además de polígonos que no representaban como tal una edificación, sino paredes individuales $\mathrm{u}$ otros objetos.

Además, se generó una digitalización de lo que respecta a la zona de La Carpio, partiendo de huellas constructivas incluídas en Open Street Map y completando el resto de forma manual. El producto final consistió en 3759 huellas para este barrio, las cuales fueron agregadas a la capa shape original de la MSJ.

\section{Tratamiento de fotos aéreas y ortofotos}

El procedimiento utilizado, que es la base para la datación de los años pertenecientes a las edificaciones, se fundamentó en el análisis de fotos aéreas para los años de 1945, 1963, 1980, 1989 y ortofotos de los años, 2005 y 2014.

Con respecto al tratamiento dado a las fotos aéreas, constituyó en la georeferenciación de cada imagen. En muchos casos, datos raster como lo son las fotos aéreas, no poseen ningún Sistema de Referencia Espacial (SRE), por lo que su geometría, es decir, su plano de ubicación, será distinta al resto de los datos que sí posean un SRE, por lo que por medio de la georeferenciación se les asigna las coordenadas correspondientes (Lizano, 2017, p. 37).

Importante mencionar que, las fotos no se ortorectificaron, al no contarse con los datos correspondientes de las cámaras con las que se realizaron los vuelos. El proceso de georeferenciación no fue necesario realizarlo con las ortofotos de los años 2005 y 2014 porque estos productos ya poseen una georeferenciación y ya han sido corregidas sus distorsiones generadas por la inclinación de las cámaras, al proyectar las imágenes de forma ortogonal, y colocando el mosaico en una misma escala (Gutiérrez, 2005, p. 144).

Una vez georreferenciadas y luego de cortar los lados adyacentes de las imágenes porque se reflejan en datos sin coordenadas en forma de bordes oscuros, se obtiene un mosaico lineal como se observa en la figura 2 , de las fotos que forman al cantón. Sobre las fotos, se coloca la capa shape de edificaciones y por medio de una fotointerpretación, es decir, de una observación y análisis de la información fotográfica, se datan los años 
Silvia Blanco-Sáenz, Luis Carlos Esquivel-Salas

Estimación del periodo de construcción de las edificaciones del cantón de San José

por medio de análisis de fotografías aéreas

de construcción. También en la figura 3 se ve reflejada una de las uniones entre las fotos, que se caracteriza por el cambio en la luz.

Figura 2. Mosaico de fotos aéreas 1980.

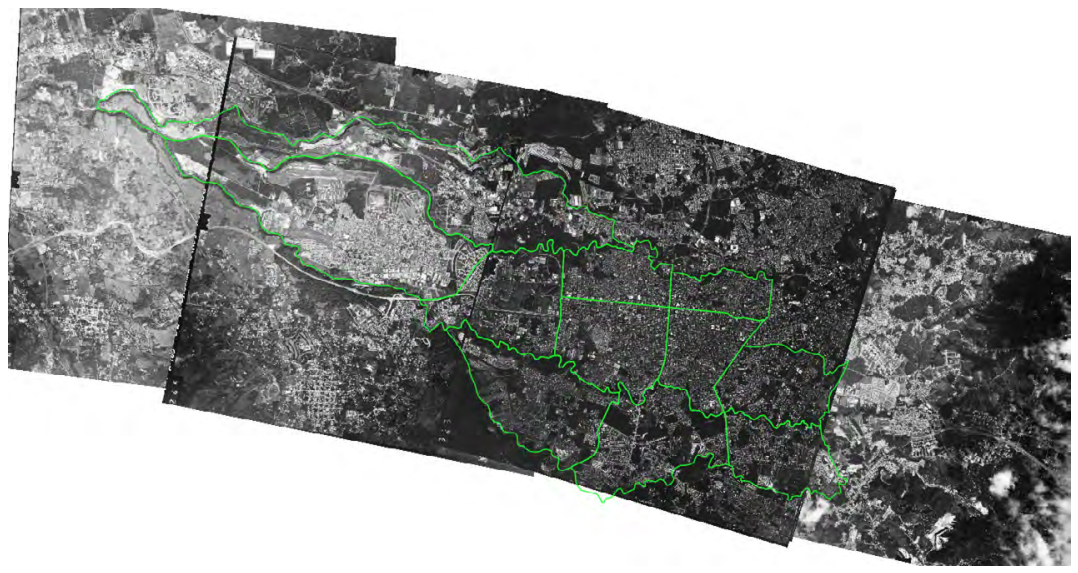

Figura 3. Mosaico de fotos aéreas 1980.

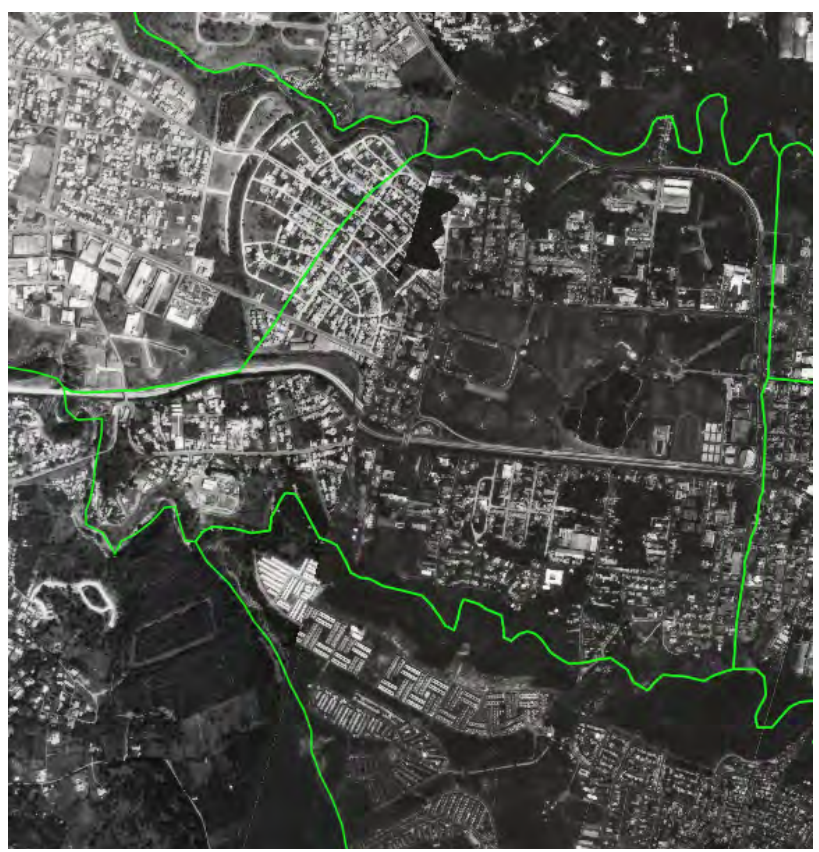


Silvia Blanco-Sáenz, Luis Carlos Esquivel-Salas Estimation of the construction period of the buildings (construction period) in the canton of San José by analysis of aerial photographs

\section{Clasificación de períodos de construcción}

El proceso de clasificación de periodos de construcción es manual, consistiendo en el análisis visual de las áreas por cada año correspondiente a la fotografía aérea $u$ ortofoto y seleccionar los polígonos que hacen referencia a edificaciones halladas en el respectivo período.

Es importante según se cambian los periodos de las fotos, que la capa shape se encuentre correctamente categorizada para que los polígonos previamente datados no sean confundidos ni equivocadamente re-datados con polígonos aún sin clasificar, para finalmente obtener un producto como el de la figura 4, en el que se observan polígonos con su adecuado periodo de construcción, que corresponden a los periodos antes de 1944, 1945 a 1962 , 1963 a 1973, 1974 a 1979, 1980 a 1988, 1989 a 2004, 2005 a 2013 y 2014 a 2017. Su respectiva representación se muestra en la figura 4.

Figura 4. Recuadro del distrito de Mata Redonda y simbología de los períodos de construcción, ortofoto, 2017.

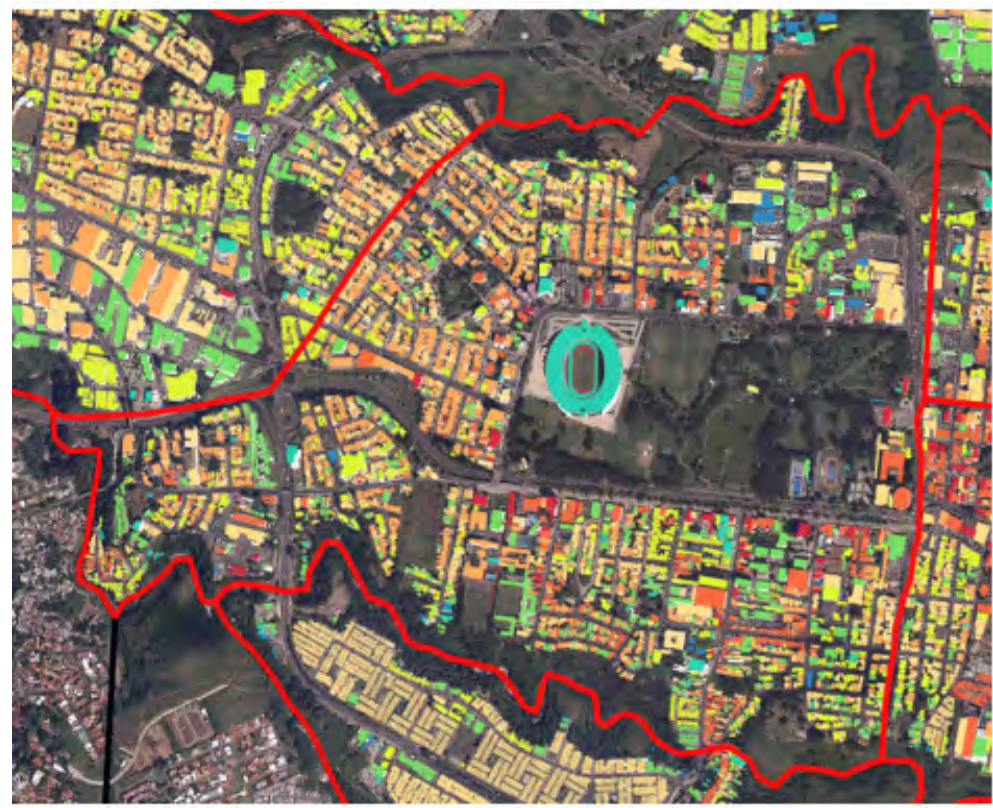




\section{Asignación de la versión de Código Sísmico de Costa Rica}

Una vez que se clasificaron las edificaciones por periodos de construcción, se procedió a asignar una versión del Código Sísmico de Costa Rica (CSCR) aplicable basado en este. Con ello la información generada es directamente utilizable y aplicable a la evaluación de riesgo sísmico del cantón de San José en desarrollo por el LIS-UCR.

Costa Rica cuenta con cuatro versiones de código sísmico, los cuales son la guía para un adecuado diseño y construcción de obras civiles, estos fueron publicados en los años 1974, 1986, 2002, 2010, y el último posee una actualización realizada en el año 2014 (Villalobos, 2020). Los años de publicación de los códigos sísmicos fueron la primera opción para la definición de los periodos de construcción a utilizar en este trabajo, más el IGN no contaba con fotos completas del cantón para los mismos, por lo que los años utilizados eran los más cercanos a estos y contaban con fotografías que cubrían en su totalidad el cantón.

La asignación de la versión código sísmico se realizó según lo mostrado en la tabla 2, que se muestra a continuación:

Tabla 2. Asignación de código sísmico.

\begin{tabular}{|c|c|c|c|}
\hline \multirow{2}{*}{ ID } & \multicolumn{2}{|c|}{ Período de construcción } & \multirow{2}{*}{ Versión del CSCR aplicable } \\
\cline { 2 - 3 } & Año inicial & Año final & Pre - Código \\
\hline $\mathbf{1}$ & -- & 1944 & Pre - Código \\
\hline $\mathbf{2}$ & 1945 & 1962 & Pre - Código \\
\hline $\mathbf{3}$ & 1963 & 1973 & 1974 \\
\hline $\mathbf{4}$ & 1974 & 1979 & 1974 \\
\hline $\mathbf{5}$ & 1980 & 1988 & 1986 \\
\hline $\mathbf{6}$ & 1989 & 2004 & 2002 \\
\hline $\mathbf{7}$ & 2005 & 2013 & 2010 \\
\hline $\mathbf{8}$ & 2014 & 2017 & \\
\hline
\end{tabular}

\section{Resultados y análisis}

Los resultados presentados se generan a partir de los datos obtenidos siguiendo la metodología anteriormente descrita. Se debe tener claro que, aunque para la mayoría de estructuras se considera que el periodo de construcción asignado es acertado, hay dos aspectos que la metodología no incluye debido a que las 84004 edificaciones se observaron desde un plano aéreo: (1) las estructuras que han sido adecuadas sísmicamente para cumplir con versiones modernas del Código Sísmico de Costa Rica y (2) 
las edificaciones demolidas y que en su lugar se construyeron otras cumpliendo normas constructivas más recientes. Por esta razón, se deben tener en cuenta estas limitaciones a la hora de analizar los resultados.

\section{Rendimientos}

El tiempo que se tardó en la estimación del periodo de construcción para las 84000 edificaciones es relevante para poder hacer proyecciones de su duración en otros estudios semejantes, ya que debe ser realizable dentro del periodo de desarrollo de una evaluación de riesgo sísmico físico.

Para la obtención, revisión y producción de los datos fueron necesarias alrededor de cuatrocientas horas de trabajo. Dependiendo de la resolución y claridad de las fotos aéreas la respectiva georeferenciación sería más ajustada a la realidad, para los primeros años se necesitó hacerla dos o más veces por la dificultad de ajustar la foto a la capa de polígonos, en promedio por foto este procedimiento tomaba de 20 a 30 minutos según la facilidad de hallar puntos de control, ya sean edificios, intersecciones o esquinas.

Con respecto a la clasificación del año de construcción, para algunas imágenes fotográficas el error residual que resultaba de la georreferenciación no se lograba disminuir lo suficiente por lo que era necesario mover bloques de polígonos y acomodarlos sobre su respectivo bloque estructural en la foto, de esta forma facilitar y reducir los errores de posibles inadecuadas clasificaciones temporales. Al tener que realizar tales movimientos, en muchos casos con miles de polígonos, se genera un evidente aumento de trabajo no observado cuando se utilizan las ortofotos, por lo que en promedio por mosaico de fotos aéreas eran necesarias aproximadamente entre 15 y 20 horas de trabajo para la calificación.

Importante tomar en cuenta los diferentes obstáculos que se presentan con la dificultad de la visualización por baja resolución de las fotos más antiguas y también que no todos los mosaicos poseen la misma cantidad de fotos aéreas. Por ejemplo, las fotos aéreas de 1945 fueron las más difíciles de georeferenciar y clasificar debido a la escala y baja resolución, mas eran solo tres fotos. Sin embargo, para el año 1989 el mosaico constaba de seis fotos pero la existencia de edificaciones clasificadas y la localización de otros puntos de referencia, junto con una escala que permitía una mayor visualización, facilitó la clasificación de polígonos para ese período de construcción. 
Silvia Blanco-Sáenz, Luis Carlos Esquivel-Salas

Estimación del periodo de construcción de las edificaciones del cantón de San José por medio de análisis de fotografias aéreas

\section{Expansión de la mancha urbana}

En la figura 5 se muestra un mapa que representa el crecimiento urbanístico generado desde las infraestructuras construidas antes de 1944 hasta el año 1962, con una cantidad de 17660 inmuebles a los largo del cantón, correspondientes al 21\% de total de edificaciones. Para 1944 los cuatro distritos más poblados eran la Merced, el Carmen, Hospital y Catedral, siendo Hospital el más urbanizado con 1282 estructura, para el periodo de periodo de 1945 a 1692 que es la segunta etapa más antigua analizada en la investigación, se construyeron otros 12863 inmuebles, que representan un 15.3\% del total cantonal.

La figura 5 permite comprobar cuáles espacios territoriales fueron los primeros en empezar a habitarse y urbanizarse, los cuatro distritos se mantienen y continúa siendo el mayor poblado el distrito de Hospital con 3091 edificaciones nuevas, para un total de alrededor de 4 373. Los distritos de la Uruca y Pavas, fueron los que más tardaron en iniciar un proceso de urbanización, pues solo para el periodo de 1945 a 1962 ambos contaban con un total de 282 construcciones.

Figura 5. Mapa períodos "antes de 1944" y 1945-1962.

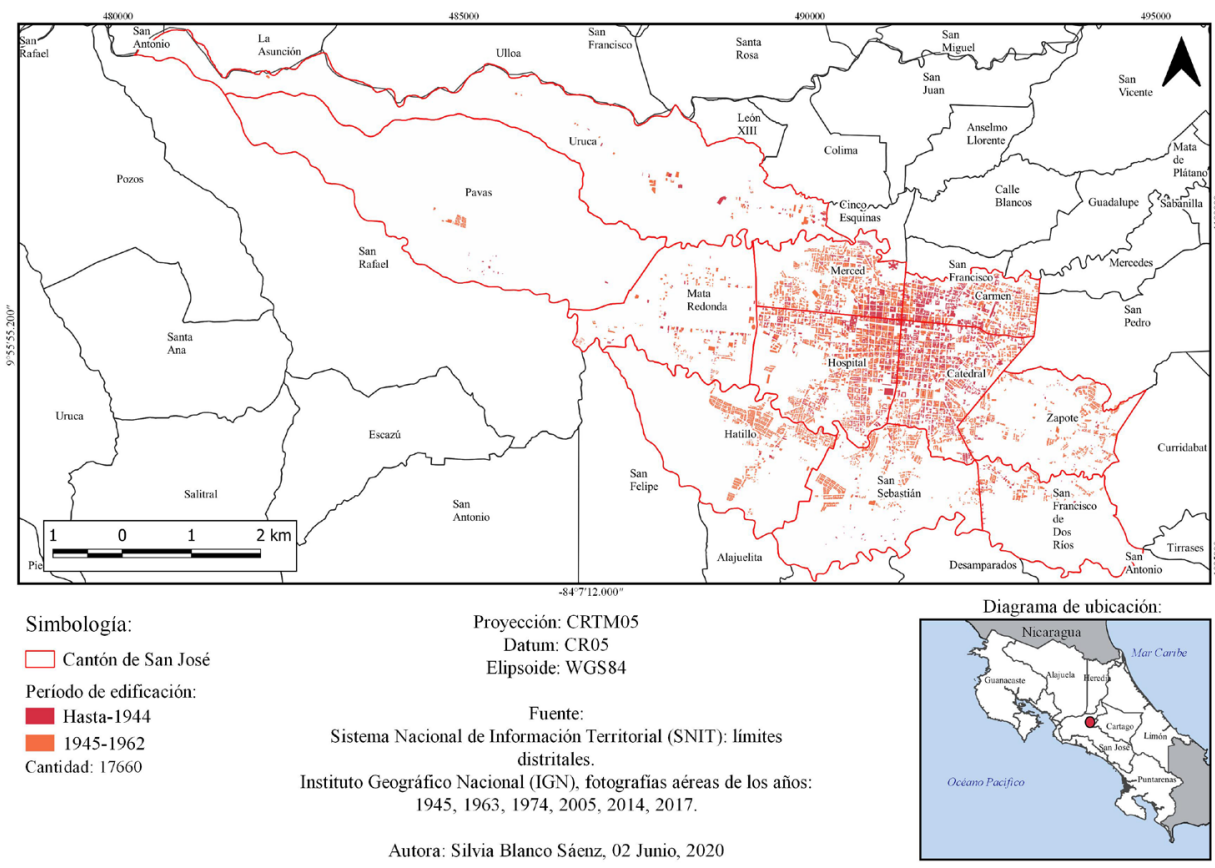


La figura 6 es un mapa que contiene las 84004 edificaciones que conforman el cantón de San José para el año 2017, clasificadas según el periodo de construcción, para ese momento el distrito con mayor cantidad de edificios es el de Pavas con aproximadamente 19685 estructuras. Importante destacar que como se mencionó en el párrafo anterior, este fue uno de los más tardíos en desarrollarse a nivel estructural pero esto se debe a que es en kilómetros cuadrados $\left(9.34 \mathrm{~km}^{2}\right)$ el distrito más grande y representa un $20.93 \%$ del total de territorio (MSJ, 2011, p.12), el distrito de Hatillo es el siguiente con 12895 inmuebles en un espacio de $4.27 \mathrm{~km}^{2}$.

Figura 6. Mapa edificaciones 1944-2017.

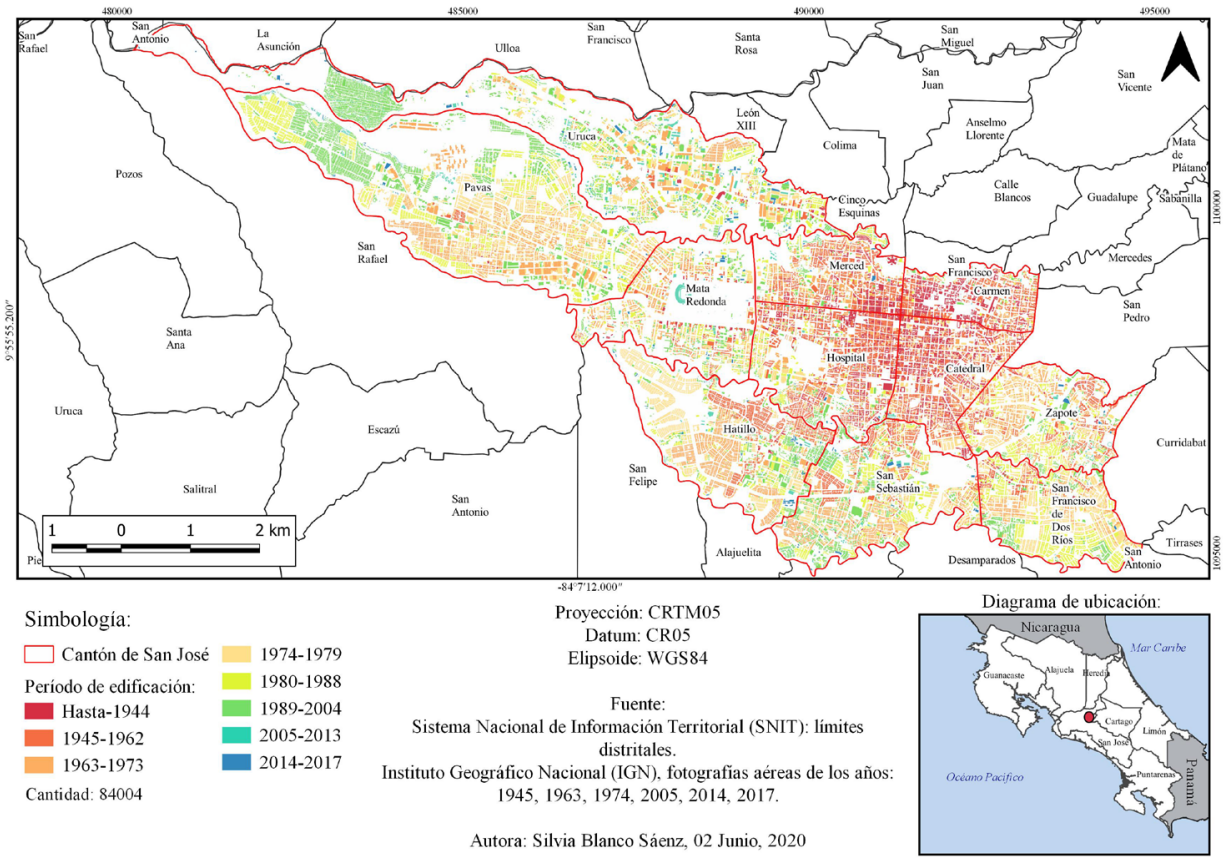

Es significativo mencionar que para el censo del año 2011 Pavas contenía al $32.1 \%$ de la población cantonal y sumando al distrito de la Uruca, San Sebastián y Hatillo albergan alrededor del 70\% de la misma. Algo que también hay que destacar es el hecho de que como ya se mencionó Pavas no solamente es el distrito con mayor población sino 
que es el más grande y por ese mismo hecho se hallan la mayor cantidad de estructuras, pero el siguiente en expansión territorial es la Uruca con $8.35 \mathrm{~km} 2$, y este espacio alberga solo un $15.2 \%$ de la población siendo el cuarto más poblado y el cuarto en cantidad de estructuras con un aproximado de 9101 inmuebles, lo previo se puede deber a que este es un distrito industrial.

También se halla que los distritos que fueron los primeros en ser poblados como la Merced, el Carmen, Hospital y Catedral, son los que menor cantidad de estructuras poseen en la actualidad, junto con Mata Redonda, siendo el distrito del Carmen el que menos cantidad detenta en todo el cantón con alrededor de 1802 edificios. Esto se puede deber a que son espacios donde la mancha urbana no puede avanzar más debido a que no hay espacios terrestres para continuar su expansión.

\section{Relacionado al Código Sísmico de Costa Rica}

Se tiene que el 38\% de la edificaciones del cantón de San José fueron construidas previo a la existencia de cualquier Código Sísmico de Costa Rica (CSCR), es decir antes del año 1974, como se observa en el gráfico 1 y tabla 3 . Además, el $60 \%$ de las edificaciones se construyeron utilizando las versiones del CSCR de los años 74 y 86, previos a la versión del 2002, donde se incluyeron importantes requisitos para aumentar la ductilidad en las uniones y regiones de rótulas plásticas, dejando solamente un $2 \%$ de las estructuras construidas con las versiones más recientes del CSCR, 2002 y 2010.

Asimismo, con respecto a las proporciones de construcción se analiza que los periodos con menor construcción corresponde a los años actuales, pues desde el 2005 el cantón solo ha experimentado el crecimiento de un $2.5 \%$ aproximadamente. La mayor etapa de expansión urbana del cantón fue en el periodo de 1989 al 2004, con un aumento de estructuras de $21.9 \%$ que en cantidad de estructuras se traducen en 18399 inmuebles, como se observa en la tabla 3 . 
Grafico 1. Porcentaje de edificaciones por periodo de construcción

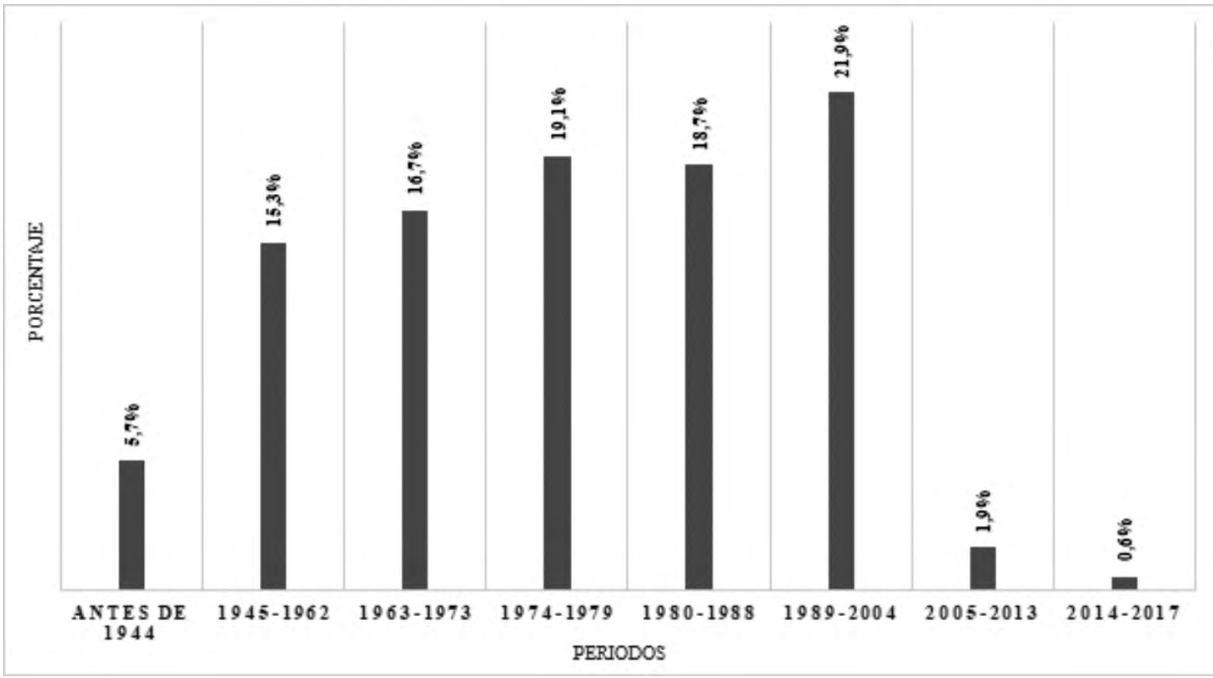

Tabla 3. Resultados de cantidad de estructuras construidas según periodo de construcción.

\begin{tabular}{|c|c|c|c|c|c|c|c|c|c|}
\hline Períodos & $\begin{array}{c}\text { Antes } \\
\text { de } 1944\end{array}$ & $\begin{array}{c}1945- \\
1962 \\
\end{array}$ & $\begin{array}{c}1963- \\
1973 \\
\end{array}$ & $\begin{array}{c}1974- \\
1979 \\
\end{array}$ & $\begin{array}{c}1980- \\
1988 \\
\end{array}$ & $\begin{array}{c}1989- \\
2004\end{array}$ & $\begin{array}{c}2005- \\
2013\end{array}$ & $\begin{array}{c}2014- \\
2017 \\
\end{array}$ & Total: \\
\hline $\begin{array}{l}\text { Cantidad de } \\
\text { estructuras }\end{array}$ & 4767 & 12893 & 14040 & 16048 & 15683 & 18399 & 1680 & 494 & 84004 \\
\hline $\begin{array}{c}\text { Porcentaje de } \\
\text { estructuras }\end{array}$ & $5.7 \%$ & $15.3 \%$ & $16.7 \%$ & $19.1 \%$ & $18.7 \%$ & $21.9 \%$ & $1.9 \%$ & $0.6 \%$ & $100 \%$ \\
\hline $\begin{array}{c}\text { Tasa de } \\
\text { construcciones } \\
\text { promedio por año }\end{array}$ & -- & 716 & 1276 & 2675 & 1743 & 1150 & 187 & 124 & -- \\
\hline $\begin{array}{c}\text { Códigos sísmicos } \\
\text { (CSCR) }\end{array}$ & \multicolumn{3}{|c|}{ Pre - Código } & \multicolumn{2}{|c|}{1974} & 1986 & 2002 & 2010 & Total \\
\hline $\begin{array}{l}\text { Total de edificaciones } \\
\text { por versión de CSCR }\end{array}$ & \multicolumn{3}{|c|}{31700} & \multicolumn{2}{|c|}{31731} & 18399 & 1680 & 494 & 84004 \\
\hline Porcentaje & \multicolumn{3}{|c|}{$37.7 \%$} & \multicolumn{2}{|c|}{$37.8 \%$} & $21.9 \%$ & $1.9 \%$ & $0.6 \%$ & $100 \%$ \\
\hline
\end{tabular}

\section{Validación de resultados}

Como un medio de validación o contraste de resultados, se buscó en la literatura las fechas de fundación de barrios de San José. Este tipo de información resultó ser difícil de encontrar, por lo que se obtuvo para 40 barrios de distintos distritos. En la tabla 4 se resume esta información. 
Silvia Blanco-Sáenz, Luis Carlos Esquivel-Salas

Estimación del periodo de construcción de las edificaciones del cantón de San José por medio de análisis de fotografias aéreas

Tabla 4. Contraste de periodos de construcción y fechas de fundación o expansión urbana de 40 barrios de San José.

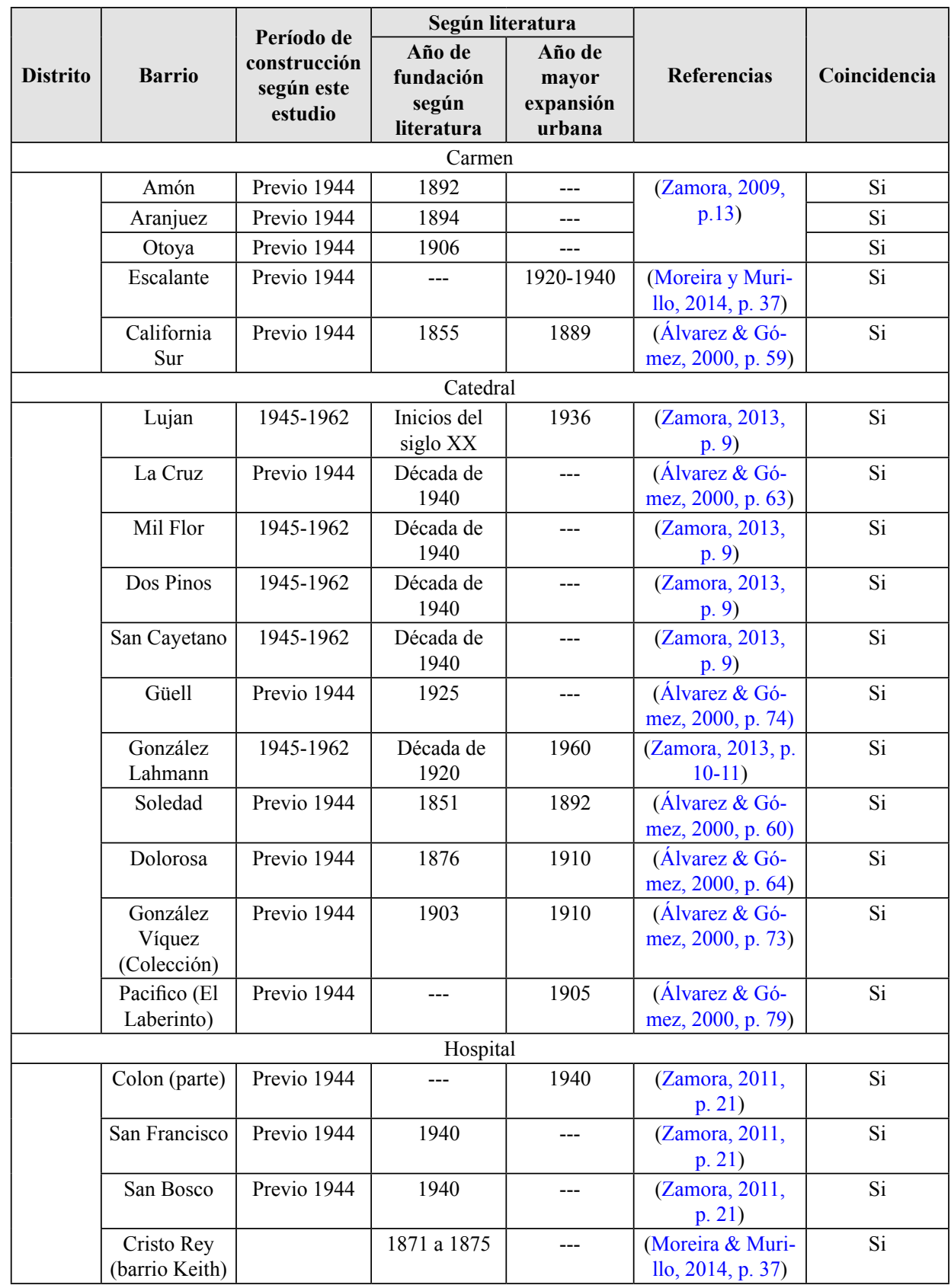


Silvia Blanco-Sáenz, Luis Carlos Esquivel-Salas Estimation of the construction period of the buildings (construction period) in the canton of San José by analysis of aerial photographs

\begin{tabular}{|c|c|c|c|c|c|c|}
\hline \multirow[b]{2}{*}{ Distrito } & \multirow[b]{2}{*}{ Barrio } & \multirow[b]{2}{*}{$\begin{array}{l}\text { Período de } \\
\text { construcción } \\
\text { según este } \\
\text { estudio }\end{array}$} & \multicolumn{2}{|c|}{ Según literatura } & \multirow[b]{2}{*}{ Referencias } & \multirow[b]{2}{*}{ Coincidencia } \\
\hline & & & $\begin{array}{l}\text { Año de } \\
\text { fundación } \\
\text { según } \\
\text { literatura }\end{array}$ & $\begin{array}{c}\text { Año de } \\
\text { mayor } \\
\text { expansión } \\
\text { urbana }\end{array}$ & & \\
\hline \multicolumn{7}{|c|}{ Merced } \\
\hline & Pitahaya & $1945-1962$ & --- & 1951 a 1955 & $\begin{array}{c}\text { (Zamora, 2011, } \\
\text { p. 19) }\end{array}$ & $\mathrm{Si}$ \\
\hline \multicolumn{7}{|c|}{ Hatillo } \\
\hline & $\begin{array}{c}15 \text { de Se- } \\
\text { tiembre } \\
\text { (Ciudadela o } \\
\text { Colonia } 15 \text { de } \\
\text { Setiembre) }\end{array}$ & $1945-1962$ & $\begin{array}{c}\text { Década de } \\
1960\end{array}$ & --- & (Blanco, 2015) & $\mathrm{Si}$ \\
\hline \multicolumn{7}{|c|}{ La Uruca } \\
\hline & La Carpio & $1989-2004$ & 1993 у 1994 & --- & $\begin{array}{l}\text { (Rojas, } 2009, \\
\text { p. } 35)\end{array}$ & $\mathrm{Si}$ \\
\hline \multicolumn{7}{|c|}{ San Sebastián } \\
\hline & Paso Ancho & Previo 1944 & 1914 & 1940 & (Valverde, 2018) & $\mathrm{Si}$ \\
\hline & Santa Rosa & $1963-1973$ & 1952 & 1964 & (Valverde, 2018) & $\mathrm{Si}$ \\
\hline \multicolumn{7}{|c|}{ Zapote } \\
\hline & $\begin{array}{l}\text { Quesada } \\
\text { Duran }\end{array}$ & $1945-1962$ & 1890 & 1940 & $\begin{array}{l}\text { (Montero. A, y } \\
\text { Marín. J, 1995, } \\
\text { p.25) }\end{array}$ & No \\
\hline & Córdoba & $1945-1962$ & 1890 & 1940 & $\begin{array}{l}\text { (Montero. A, y } \\
\text { Marín. J, 1995, } \\
\text { p.25) }\end{array}$ & No \\
\hline & $\begin{array}{l}\text { Moreno } \\
\text { Cañas }\end{array}$ & $1945-1962$ & 1950 & --- & $\begin{array}{l}\text { (Montero. A, y } \\
\text { Marin. J, 1995, } \\
\text { p.28) }\end{array}$ & $\mathrm{Si}$ \\
\hline & La Gloria & $1945-1962$ & --- & 1957 & $\begin{array}{l}\text { (Montero. A, y } \\
\text { Marín. J, 1995, } \\
\text { p.35) }\end{array}$ & $\mathrm{Si}$ \\
\hline & Las Luisas & $1963-1973$ & 1960 & --- & $\begin{array}{l}\text { (Montero. A, y } \\
\text { Marín. J, 1995, } \\
\text { p.35) }\end{array}$ & $\mathrm{Si}$ \\
\hline & Trébol & 1974-1979 & 1960 & --- & $\begin{array}{l}\text { (Montero. A, y } \\
\text { Marín. J, 1995, } \\
\text { p.35) }\end{array}$ & No \\
\hline & Yoses Sur & $1963-1973$ & 1960 & --- & $\begin{array}{l}\text { (Montero. A, y } \\
\text { Marín. J, 1995, } \\
\text { p.35) }\end{array}$ & $\mathrm{Si}$ \\
\hline & San Dimas & $1963-1973$ & 1960 & --- & $\begin{array}{l}\text { (Montero. A, y } \\
\text { Marín. J, 1995, } \\
\text { p.35) }\end{array}$ & $\mathrm{Si}$ \\
\hline
\end{tabular}


Silvia Blanco-Sáenz, Luis Carlos Esquivel-Salas

Estimación del periodo de construcción de las edificaciones del cantón de San José por medio de análisis de fotografias aéreas

\begin{tabular}{|c|c|c|c|c|c|c|}
\hline \multirow[b]{2}{*}{ Distrito } & \multirow[b]{2}{*}{ Barrio } & \multirow[b]{2}{*}{$\begin{array}{l}\text { Período de } \\
\text { construcción } \\
\text { según este } \\
\text { estudio }\end{array}$} & \multicolumn{2}{|c|}{ Según literatura } & \multirow[b]{2}{*}{ Referencias } & \multirow[b]{2}{*}{ Coincidencia } \\
\hline & & & $\begin{array}{c}\text { Año de } \\
\text { fundación } \\
\text { según } \\
\text { literatura }\end{array}$ & $\begin{array}{l}\text { Año de } \\
\text { mayor } \\
\text { expansión } \\
\text { urbana }\end{array}$ & & \\
\hline \multicolumn{7}{|c|}{ Pavas } \\
\hline & Bribri & $1980-1988$ & 1984 & --- & $\begin{array}{c}\text { (Vargas. M, 2013, } \\
\text { p. } 22)\end{array}$ & $\mathrm{Si}$ \\
\hline & Metrópolis & 1989-2004 & 1988 & -- & $\begin{array}{c}\text { (Vargas. M, 2013, } \\
\text { p. } 22)\end{array}$ & $\mathrm{Si}$ \\
\hline & San Pedro & $1989-2004$ & 1987 & --- & $\begin{array}{c}\text { (Vargas. M, 2013, } \\
\text { p. 23) }\end{array}$ & $\mathrm{Si}$ \\
\hline \multicolumn{7}{|c|}{ Mata Redonda } \\
\hline & Colon (parte) & Previo 1944 & --- & 1940 & $\begin{array}{c}\text { (Zamora, 2011, } \\
\text { p. 21) }\end{array}$ & $\mathrm{Si}$ \\
\hline & Sabana & Previo 1944 & 1894 & 1915 & (MSJ, s.f.) & $\mathrm{Si}$ \\
\hline & Rohrmoser & $1963-1973$ & 1851 & --- & $\begin{array}{l}\text { (Hilje., } 2010, \\
\text { p. 29) }\end{array}$ & No \\
\hline \multicolumn{7}{|c|}{ San Francisco de Dos Ríos } \\
\hline & San Francisco & 1963-1973 & 1801 & 1950 & (MSJ, s.f.) & No \\
\hline
\end{tabular}

De los 40 barrios a los cuales se logró encontrar una fecha de fundación en literatura, solamente en cinco de ellos no hubo coincidencia entre esta y el período de construcción asignado en este estudio. De ellos, dos tienen una diferencia de apenas cinco años, mientras en los tres se presentan diferencias de mayor cantidad de años.

Es importante aclarar que las razones por las cuales en algunos de los barrios no hay una compaginación entre fecha de clasificación por foto aérea y fecha bibliográfica puede deberse a aspectos como que se dató la fecha de llegada de la familia fundadora como lo que en el caso del barrio Rohrmoser, o primeros arribos como en los barrios de Trébol y San Francisco, más se desconoce de posibles procesos de migración que llevaron a un aumento de población por ende de la edificación de asentamientos.

Quesada Dura y Córdoba también presentan desplazamiento temporal, de aproximadamente cinco años, lo cual no es una disidencia muy amplia pues los datos bibliográficos pueden haberse enfocado en asentamientos iniciales a la hora de datar las fechas de fundamentación y consolidación ignorado otro procesos de movimiento poblacional/ comercial. 


\section{Conclusiones}

Se presenta un estudio que consistió en la estimación de periodos de construcción aproximados para las 84000 edificaciones del cantón de San José por medio del análisis de fotografías aéreas y ortofotos. Se demostró que estos insumos, disponible en el Instituto Geográfico Nacional (IGN) de Costa Rica, pueden ser utilizados con este fin, logrando un nivel de precisión adecuado tomando en cuenta la cantidad de estructuras trabajadas. Así mismo, se documentó la duración del proceso para posibilitar proyecciones para otros estudios similares.

El período con mayor cantidad de estructuras desarrolladas fue el de 1989-2004, en el que se construyeron 18399 estructuras, representando un $22 \%$ del total de edificaciones presentes al 2017. El periodo con menor edificaciones construidas fue el de 2014-2017, con 494 estructuras representado solamente un $0.6 \%$. Se calculó una tasa de construcción promedio, la cual tuvo su valor más alto para el periodo de 1974-1979, con 2675 edificaciones construidas por año, seguida del periodo 1980-1988 con 1743.

El 38\% de las edificaciones se construyeron previo a la existencia del Código Sísmico de Costa Rica (CSCR), el 60\% utilizando las versiones del CSCR de los años 1974 y 1986 . Solamente un 2\% de las estructuras corresponden a las versiones más recientes del CSCR, 2002 y 2010, las cuales incluyeron requisitos esenciales para la seguridad de la edificación, como lo serían el aumento de la ductilidad en las uniones y regiones de rótulas plásticas.

Se hace un contraste de los resultados obtenidos con fuentes bibliográficas, logrando obtener información para 40 de los 196 barrios que componen el cantón de San José. Solamente en cinco de ellos no hubo coincidencia entre el periodo de construcción determinado por medio de la fuente bibliográfica y el asignado en este estudio. De ellos, dos tienen una diferencia de apenas cinco años, mientras en los tres se presentan diferencias de mayor cantidad de años. Esto se considera una medida de la precisión de los resultados, siendo bastante aceptable.

Finalmente, el dato del periodo de construcción para edificaciones puede ser útil para distintos fines, entre ellos los estudios de riesgo ante amenazas naturales, no limitándose exclusivamente a la amenaza sísmica. En este trabajo se expone su gran valor y utilidad en el contexto de evaluaciones de riesgo sísmico físico. 
Silvia Blanco-Sáenz, Luis Carlos Esquivel-Salas

Estimación del periodo de construcción de las edificaciones del cantón de San José

por medio de análisis de fotografias aéreas

\section{Financiamiento}

Esta investigación se financió con fondos provenientes del Transitorio I de la Ley Nacional de Emergencias ( $N^{\circ}$ 8488) de la República de Costa Rica.

\section{Reconocimientos}

A los estudiantes de la Escuela de Geografía de la Universidad de Costa Rica: Brayna Ruiz, quien colaboró con el proyecto en el año 2018 y generó los polígonos de las huellas constructivas del barrio de La Carpio; Kevin Cordero, quien colaboró durante el periodo 2020 en la clasificación de años de construcción.

\section{Referencias}

Álvarez, Y. y Gómez, D. (2000). San José de Antaño, Distrito Catedral (1890-1940). Ministerio de Cultura y Juventud: Centro de Investigación y Conservación del Patrimonio Cultural. http://www.patrimonio. go.cr/biblioteca_digital/publicaciones/San_Jose_de_Antano.html Blanco, R. (2015). "Los del Sur de la Ciudad Capital": Control Social y Estigmatización en los Barrios del Sur de San José, 1950-1980. Diálogos Revista Electrónica. Vol. 16 (2). https://revistas.ucr.ac.cr/ index.php/dialogos/article/view/17880/19531

Calderón, A. (2016). Probabilistic Earthquake Loss Assessment for Costa Rica. In Masters Thesis. Earthquake and Seismology Engineering. Instituto Universitario di Studi Superiori di Pavia.

Calderón, A., \& Silva, V. (2019). Probabilistic seismic vulnerability and loss assessment of the residential building stock in Costa Rica. $\mathrm{Bu}$ lletin of Earthquake Engineering, 17(3), pp. 1257-1284. https://doi. org/10.1007/s10518-018-0499-1

Cardona, O., Yamín, L., Bernal, G., Ordaz, M., Reinoso, E., Barbat, A., \& Carreño, M. (2009a). T2-2 Recursos de información disponibles. Informe de CAPRA Costa Rica, pp. 39. https://www.ecapra.org/ documentos/documentos-capra-costa-rica

Cardona, O., Yamín, L., Bernal, G., Ordaz, M., Reinoso, E., Barbat, A., \& Carreño, M. (2009b). T2-3 Modelación probabilista de amenazas naturales. Informe de CAPRA Costa Rica, pp. 93. https://www.ecapra. org/documentos/documentos-capra-costa-rica 
Cardona, O., Yamín, L., Bernal, G., Ordaz, M., Reinoso, E., Barbat, A., \& Carreño, M. (2009c). T2-4 Inventario de elementos expuestos. Informe de CAPRA Costa Rica, pp. 1-42. https://www.ecapra.org/ documentos/documentos-capra-costa-rica

Cardona, O., Yamín, L., Bernal, G., Ordaz, M., Reinoso, E., Barbat, A., \& Carreño, M. (2009d). T2-5 Caracterización local de la vulnerabilidad de edificaciones. Informe de CAPRA Costa Rica, pp. 1-66. https://www.ecapra.org/documentos/documentos-capra-costa-rica

Esquivel-Salas, L. (2020). Avance del modelo de exposición del cantón de San José para evaluaciones de riesgo sísmico. Revista Ingeniería, 30(2), pp. 103-119. https://doi.org/10.15517/RI.V30I2.40469

Evaluación de Riesgos Naturales y Antropogénicos. (2014). Valoración de la vulnerabilidad física y riesgo ante sismos en distritos clave del Cantón de San José. ERN, 153.

FEMA. (2013). Hazus-MH 2.1: Earthquake Model Technical Manual. In FEMA. www.fema.gov/plan/prevent/hazus

Gutiérrez, J. (2005). Topografía para las Tropas. Coronel (I.P.M): $\mathrm{N}^{\circ}$ 149.227. Consultado el 26 de mayo de 2020, de http://cartomap.cl/ utfsm/Texto-Topograf\%EDa/Cap\%2009\%20Ortofoto.pdf

Hilje, L. (2010). La Vida En San Josea Mediados Del Siglo Xix.Remembranzas De Don Chico Rohrmoser. Revista Herencia Vol. 23 (2), pp. 29-30, https://maritzacartin.files.wordpress.com/2019/01/la-vidaen-sj-a-mediados-del-siglo-xix-chico-rohmoser.pdf

Lizano, M. (2017). Curso de Sistemas de Información Geográfica utilizando QGIS.

Miyamoto Internacional Inc. (2016). Assessment of Earthquakes Risks. The USAID / OFDA PREPARE Program. Phase I., December, pp. 1-59.

Montero. A. y Marin. J, (1995). Zapote y su Historia. Universidad de Costa Rica.

Moreira, J., y Murillo, P. (2014). Convivencia urbana en San José. Un estudio sobre las prácticas cotidianas, las representaciones y proyecciones de las y los habitantes de barrio Cristo Rey y Escalante (2011-2013). CLACSO: CICDE-UNED. http://biblioteca.clacso. edu.ar/Costa_Rica/cicde-uned/20170628054956/pdf_858.pdf

Mouroux, P., Bertrand, E., Bour, M., Le Brun, B., Depinois, S., \& Masure, P. (2004). The European RISK-UE project: an advanced approach 
to earthquake risk scenarios. 13th World Conference on Earthquake Engineering, paper No. 3329, 14.

Municipalidad de San José (MSJ). (s.f.). Breve historia del Distrito Mata Redonda. Municipalidad de San José. Consultado el 28 de junio de 2020, de: https://www.msj.go.cr/MSJ/Capital/SiteAssets/distritos/ Bcas_historia $\% 20$ mata\%20redonda.pdf

Municipalidad de San José. (MSJ). (s.f.).Breve historia del Distrito. Municipalidad de San José. Consultado el 28 de junio de 2020, de: https:// www.msj.go.cr/MSJ/Capital/SiteAssets/distritos/Bcas_historia $\% 20$ san $\% 20$ francisco.pdf

Municipalidad de San José. (MSJ). (2011, agosto). Diagnóstico Cantonal. https:/www.msj.go.cr/informacion_ciudadana/SiteAssets/ DIAGN\%C3\%93STICO\%20CANTONAL.pdf

Obando. (2018). Comunicación Personal, 20 de mayo 2020.

QGIS. (s.f.). Acerca de QGIS. Consultado el 04 de junio de 2020. https:// www.qgis.org/es/site/about/index.html

Rojas, T. (2009). Espacios de Comercio para la Carpio: Reconquistando la Ciudad. (Tesis de Licenciatura, Universidad de Costa Rica). Repositorio Institucional UCR. http://www.kerwa.ucr.ac.cr/ handle/10669/75439

Simpson, A., Murnane, R., Saito, K., Phillips, E., Reid, R., \& Himmelfarb, A. (2014). Understanding risk. In GFDRR-World Bank. World Bank. https://doi.org/10.1136/bmj.329.7474.1086

Sistema Nacional de Información Territorial (SNIT). (s.f) Ortofoto 2015$20181 \mathrm{k}$. Consultado el 04 de junio de 2020. http://www.snitcr.go.cr/ servicios_ogc_lista_capas? $\mathrm{k}=\mathrm{bm} 9 \mathrm{kbzo} 6 \mathrm{NjA}=\&$ nombre $=$ Ortofo to $\% 202015-2018 \% 201 \mathrm{k}$

Valverde, E. (18 de enero de 2018). Cr-Paso ancho San Sebastián. Algunas fechas importantes en la historia de Paso Ancho. Caminante del Sur. Consultado 15 de mayo de 2020. https://caminantedelsur. com/2018/01/18/cr-pasoancho-san-sebastian-algunas-fechas-importantes-en-la-historia-de-paso-ancho/

Vargas, M. (2013). Una aproximación al desarrollo histórico y socioeconómico de la región de Pavas (1783-2013). Encuentro de Interculturalidad. Consultado el 28 de junio de 2020, de https://docs.google. com/file/d/0B2AJEdLISOWFMVRDNnhHUWdXMG8/edit 
Villalobos. (2020). Comunicación Personal, 27 de mayo 2020.

Zamora, C. (2009). Distrito Carmen, Cantón de San José. Ministerio de Cultura y Juventud: Centro de Investigación y Conservación del Patrimonio Cultural. http://www.patrimonio.go.cr/biblioteca_digital/ publicaciones/Circuito\%20E1\%20Carmen.html

Zamora, C. (2011). Paseo Colón (Distrito Merced y Hospital), Ciudad de San José. Ministerio de Cultura y Juventud: Centro de Investigación y Conservación del Patrimonio Cultural. http://patrimonio.go.cr/biblioteca_digital/publicaciones/Paseo $\% 20$ Colon.html

Zamora, C. (2013). Distrito Catedral, Cantón de San José. Ministerio de Cultura y Juventud: Centro de Investigación y Conservación del Patrimonio Cultural. http://patrimonio.go.cr/biblioteca_digital/publicaciones/2013/Circuito_turistico_Catedral.html 